\title{
Does level of processing affect the transition from unconscious to conscious perception?
}

\author{
Anna Anzulewicz ${ }^{\mathrm{a}, *}$, Dariusz Asanowicz ${ }^{\mathrm{a}}$, Bert Windey ${ }^{\mathrm{b}}$, Borysław Paulewicz ${ }^{\mathrm{c}}$, \\ Michał Wierzchon ${ }^{\mathrm{a}}$, Axel Cleeremans ${ }^{\mathrm{b}}$ \\ ${ }^{a}$ Consciousness Lab, Institute of Psychology, Jagiellonian University, Kraków, Poland \\ ${ }^{\mathrm{b}}$ Consciousness, Cognition and Computation Group, Université Libre de Bruxelles, Brussels, Belgium \\ ${ }^{\mathrm{c}}$ Warsaw School of Social Science and Humanities, Faculty in Katowice, Poland
}

\section{A R T I C L E I N F O}

\section{Article history:}

Received 18 August 2014

Available online 7 June 2015

\section{Keywords:}

Consciousness

Awareness

Vision

Levels of processing

Gradual

Dichotomous

\begin{abstract}
A B S T R A C T
Recently, Windey, Gevers, and Cleeremans (2013) proposed a level of processing (LoP) hypothesis claiming that the transition from unconscious to conscious perception is influenced by the level of processing imposed by task requirements. Here, we carried out two experiments to test the LoP hypothesis. In both, participants were asked to classify briefly presented pairs of letters as same or different, based either on the letters' physical features (a low-level task), or on a semantic rule (a high-level task). Stimulus awareness was measured by means of the four-point Perceptual Awareness Scale (PAS). The results showed that low or moderate stimulus visibility was reported more frequently in the low-level task than in the high-level task, suggesting that the transition from unconscious to conscious perception is more gradual in the former than in the latter. Therefore, although alternative interpretations remain possible, the results of the present study fully support the LoP hypothesis.
\end{abstract}

(c) 2015 Elsevier Inc. All rights reserved.

\section{Introduction}

Our subjective experience suggests that in some cases a visual stimulus might pop up in consciousness instantly, whereas in other cases, awareness of a stimulus might arise only gradually. Congruently, recent empirical studies on visual awareness have demonstrated either a sharp threshold beyond which initially unconscious information becomes conscious (Asplund, Fougnie, Zughni, Martin, \& Marois, 2014; Sergent \& Dehaene, 2004), or a gradual transition from unconscious to conscious processing (Nieuwenhuis \& de Kleijn, 2011; Overgaard, Rote, Mouridsen, \& Ramsøy, 2006; Sandberg, Timmermans, Overgaard, \& Cleeremans, 2010). Yet, the actual causes of these contrasting phenomena are poorly understood, and current theories of consciousness offer rather contradictory predictions in this respect. For instance, the global neuronal workspace model (Dehaene, Changeux, \& Naccache, 2011) predicts a sharp threshold for conscious perception, whereas the radical plasticity thesis (Cleeremans, 2011) supports the idea that the underlying processes are fundamentally graded. The latter view is also, at least partially, consistent with Lamme's (2010) hypothesis of recurrent neural mechanisms, which likewise assumes gradual access to conscious content.

\footnotetext{
* Corresponding author.

E-mail address: anna.anzulewicz@gmail.com (A. Anzulewicz).
} 
Recently, Windey, Gevers, and Cleeremans (2013) pointed out that less gradual or even dichotomous dynamics have typically been obtained in tasks that involve relatively complex stimuli, such as number or word categorization tasks (del Cul, Baillet, \& Dehaene, 2007; Gaillard et al., 2009), whereas the gradual pattern has typically been reported in studies that involve very simple stimuli such as gratings or shapes (Overgaard et al., 2006; Sandberg et al., 2010). Accordingly, Windey and colleagues (2013; Windey, Vermeiren, Atas, \& Cleeremans, 2014) proposed the hypothesis that level of processing (LoP), as imposed by the requirements of any specific task (cf. Craik \& Lockhart, 1972; Lockhart \& Craik, 1990; Petersen, Fox, Posner, Mintun, \& Raichle, 1988), influences the dynamics of the transition from unconscious to conscious perception. The more complex the required processing is, the less gradual, or more nonlinear, is the transition. Windey et al. (2013) tested this prediction by comparing accuracy and subjective visibility ratings in two tasks, a color identification task (a simple, "low-level" processing task) and a numerical judgment task (a more complex, "high-level" processing task), performed on the very same stimuli (colored digits). The stimuli were presented briefly (within a range of $10-80 \mathrm{~ms}$ in a psychophysical design) and masked. Stimuli were rated for subjective visibility using the four-point Perceptual Awareness Scale (PAS, Ramsøy \& Overgaard, 2004). In line with the predictions, the results indicated slightly more gradual patterns of conscious perception in the low-level than in the high-level task.

The results of Windey et al. (2013) support the LoP hypothesis, however, the study failed to present evidence that the two tasks indeed involve different levels of processing. Such evidence may be easily provided by means of straightforward mental chronometry (a method based on the subtractive method of Donders and the additive factor logic of Sternberg: see e.g., Posner, 2005). Specifically, if a task A involves some higher stages of processing than task B, then response time in task A should be longer than in Task B, because any additional stages of processing must take some additional time to be completed. If there is no such difference between two tasks or conditions, then we do not know if they indeed involve different levels of processing.

The present study aims to address this issue and to provide a further test for the LoP hypothesis. We used a classic Posner's paradigm, which has been developed to study the levels (or depth) of processing (Posner \& Mitchell, 1967). In two experiments (E1 and E2), we asked participants to classify pairs of letters as same or different, based either (1) on their physical identity (e.g., both letters are uppercase - E1, or in the same color - E2)-a factor assumed to involve low LoP, or (2) on a rule (e.g., both letters are consonants, E1 and E2)-a factor assumed to involve high LoP (cf. Petersen et al., 1988; Posner \& Mitchell, 1967). In E1 we used a letter case identity task as the low LoP task, based on the Posner and Mitchell (1967) procedure, whereas in E2 we used a color identity task, which allows for better differentiation between the low- and high-level. This is because comparing the physical identity of two letters might still be taken to involve minimal semantic processing (cf. Dehaene \& Cohen, 2011). The color identification task should minimize such involvement (and also make the procedure more comparable with Windey et al., 2013). All stimuli in both experiments were presented briefly (over eight presentation durations) and masked to ensure limited visibility. Further, in both experiments, subjective visibility of the stimuli was measured by means of the four-points PAS scale. Based on the logic of mental chronometry, we expected response times (RT) to be longer in the high-level task than in the low-level task. Following the LoP hypothesis, we expected the transition from unconscious to conscious perception to be more gradual in the low-level task than in the high-level task. A more gradual transition would be reflected in the higher frequency of middle PAS ratings ( 2 and 3 ), indicating that the stimuli are consciously perceived, but with moderate or even low visibility. On the other hand, observing that the middle ratings are rarely used (in favor of the highest 4th rating) would suggest that when the stimuli are perceived consciously, they are perceived right away with clear visibility, indicating a less gradual, or even dichotomous transition from unconscious to conscious processing (see Sandberg et al., 2010; Sergent \& Dehaene, 2004; Wierzchoń, Paulewicz, Asanowicz, Timmermans, \& Cleeremans, 2014; Windey et al., 2014, for a similar approach). Thus, in operational terms, we expected that in the high-level task the middle PAS ratings will be used less frequently as compared to the low-level task.

\section{Method}

\subsection{Participants}

Forty-two participants ( 6 males, mean age $=19.76, \mathrm{SD}=1.23)$ took part in Experiment 1, and 52 participants ( 11 males, mean age $=19.75, \mathrm{SD}=1.58)$ took part in Experiment 2. One participant in E1 was an outlier in both accuracy and PAS results and was excluded from the analyses. All participants were students of the Jagiellonian University and participated in the study in exchange for course credits. They reported normal or corrected-to-normal vision, normal color vision, and no history of neurological disorders. In both experiments, participants were randomly assigned to one of the two experimental groups performing either the low-level, or the high-level task.

\subsection{Stimuli, apparatus, and procedure}

\subsubsection{Experiment 1}

The classic paradigm developed by Posner and Mitchell (1967) was used to induce low- and high-level of processing. Pairs of Latin letters (both lower- and uppercase) presented simultaneously on the screen served as target stimuli. The uppercase letters subtended $\sim 2.0^{\circ}$ of visual angle. The letters were paired together either as physically identical (e.g., 'AA') or physically 


\section{Experiment 1}

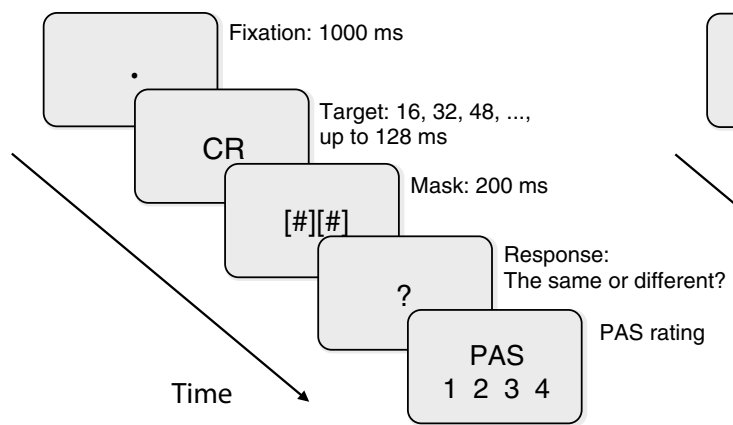

\section{Experiment 2}

Fig 1. Examples of trials from Experiment 1 (left) and Experiment 2 (right). In both experiments participants were asked to classify pairs of letters as same or different, based either on their physical identity (low-level task) or a rule (high-level task). In E1, for the low-level task, the letters were paired either as physically identical (e.g., 'AA') or physically different (e.g., 'Aa'). For the high-level task, the letters were paired as both consonants or both vowels (e.g., 'AA' or 'bb'), or as one consonant and one vowel (e.g., 'AB', or 'ec'). In E2, for the low-level task, participants were asked whether the two letters were the same in terms of their color. For the high-level, the same task as in E1 was used. Each trial started with a fixation point presented for 1000 ms, followed by the target letters presented for $16,32,48,64,80,96,112$, or $128 \mathrm{~ms}$, and by the mask presented for $200 \mathrm{~ms}$. Next, the response screen was presented, prompting participants to respond whether the letters were "the same" or "different", and then, to rate the visibility of the letters on the 4-point Perceptual Awareness Scale (PAS) scale. (For interpretation of the references to color in this figure legend, the reader is referred to the web version of this article.)

different (e.g., 'Aa') for the low-level task, and as both consonants or both vowels (e.g., 'AA' or 'bb'), or as one consonant and one vowel (e.g., 'AB', or 'ec'), for the high-level task (to avoid confusion, in the high-level task, each pair consisted either of two lowercase or two uppercase letters). Ten different combinations of letters were used in each possible task condition (i.e., ten different pairs of two physically identical capital letters, ten different pairs of two physically different uppercase letters, ten different pairs of two physically identical lowercase letters, etc.). All letters were rendered in black color. The target stimuli were followed by a mask consisting of two hash symbols flanked by square brackets: "[\#\#]". The stimuli were presented at the center of a $21^{\prime \prime}$ computer screen, with a refresh rate of $60 \mathrm{~Hz}$ (i.e., frame duration $\approx 16$ ms), via DMDX software, which allows precise control of stimulus display timing (Forster \& Forster, 2003; Garaizar, Vadillo, López-de-Ipiña, \& Matute, 2014).

An example trial is depicted in Fig. 1. On each trial, the following sequence of events took place: a central fixation cross was first presented for $1000 \mathrm{~ms}$. Next, target stimuli (a pair of letters) appeared (16-128 ms) and was immediately followed by the mask ( $200 \mathrm{~ms}$ ). Target stimuli were presented for a duration ranging from 16 to $128 \mathrm{~ms}$ ( 8 possibilities, in steps of $16 \mathrm{~ms}$ ). The order of the target durations was random. Short stimulus durations were used in order to avoid a ceiling effect in visibility ratings. After each trial, participants classified the stimuli as same or different. The low-level task required classification of the letters based on their surface characteristics, i.e., physical identity: "are both letters lowercase or uppercase?" The high-level task required classification based on semantic identity: "are both letters vowels or consonants?" Participants responded by pressing one of two color-marked keys: a green key (Enter) for the answer: "same", and a red key (right Shift) for the answer "different". The pattern of responses with the color labels appeared on the screen right after the mask offset and remained on the display until the response. Participants were instructed to guess if they could not answer or if they were uncertain about their choice. After each classification response, participants were asked to rate stimulus visibility using the four-point Perceptual Awareness Scale (PAS, Ramsøy \& Overgaard, 2004), with the points labeled as follows: 1. 'I didn't see the letters at all', 2. 'I only saw a brief glimpse of the letters', 3. 'I saw the letters fairly clearly', and 4. 'I saw the letters absolutely clearly'. The scale with labels appeared on the screen right after the identification response and remained until participants had provided the PAS response.

The entire task consisted of 640 trials ( 80 trials per condition) divided into 2 blocks, preceded by 12 training trials in which accuracy feedback was provided after each response. Participants were also informed that they would be given feedback on their overall accuracy in the test trials at the end of the task.

\subsubsection{Experiment 2}

The task used in Experiment 2 was almost identical to the one used in Experiment 1, except for a one major change: For the low-level processing, a color identity task was used instead of the letter case task, that is, participants were asked whether the two letters presented on the screen had the same color. The high-level task remained the same as in E1, where participants classified the letters based on their semantic identity. An example trial is depicted in Fig. 1.

The same stimuli were used in both, low- and high-level tasks of E2. The target letters were presented in 10 different colors (from the Apple palette). ${ }^{1}$ In both tasks, the letter pairs appeared either in the same color ( $50 \%$ of trials) or in different colors

\footnotetext{
1 The following colors were used: green - RGB 0,255,0; magenta - RGB 255,0,255; black - RGB 0,0,0; blue - RGB 0,0,255; brown - RGB 153,102,51; cyan RGB 0,255,255; orange - RGB 255,126,0; purple - RGB 127,0,127; red - RGB 255,0,0 and yellow 255,255,0).
} 
(the other $50 \%$ of trials). Letter color was irrelevant in the case of the high-level task. Each pair consisted either of two lowercase or two uppercase letters. All other parameters of stimuli, the apparatus, and the procedure remained the same as in Experiment 1 .

\section{Results}

\subsection{Classification responses}

The average response times (RT) for correct identification responses and the average accuracy for each condition of Experiment 1 and Experiment 2 are presented in Fig. 2. Response times and accuracy rates were analyzed by means of a 2 LoP (low, high; between subject factor) $\times 8$ Target Duration (16-128 ms; within subject factor) general linear model with repeated measures. Significant interactions were further analyzed by means of $t$ tests on subsets of the data. Statistical significance of Target Duration effects was corrected with the Greenhouse-Geisser or Bonferroni methods.

\subsubsection{Response time (RT)}

Experiment 1. In the high-level task, overall RT of correct responses was $280 \mathrm{~ms}$ longer than in the low-level task (1357 vs $1076 \mathrm{~ms}$, the main effect of LoP: $\left.F(1,39)=9.46, p=.004, \eta_{p}{ }^{2}=.19\right)$. The main effect of Target Duration was also significant, $F(7,273)=72.30, p<.0001, \eta_{p}{ }^{2}=.65$. The LoP $\times$ Target Duration interaction was not significant, $F<1.0$.

Experiment 2. A similar effect of longer RT for correct responses in the high-level task was observed in E2 for each target duration, except the $16 \mathrm{~ms}$ duration, due to a floor effect in this condition (the accuracy was hardly above the chance level,

\section{Experiment 1}

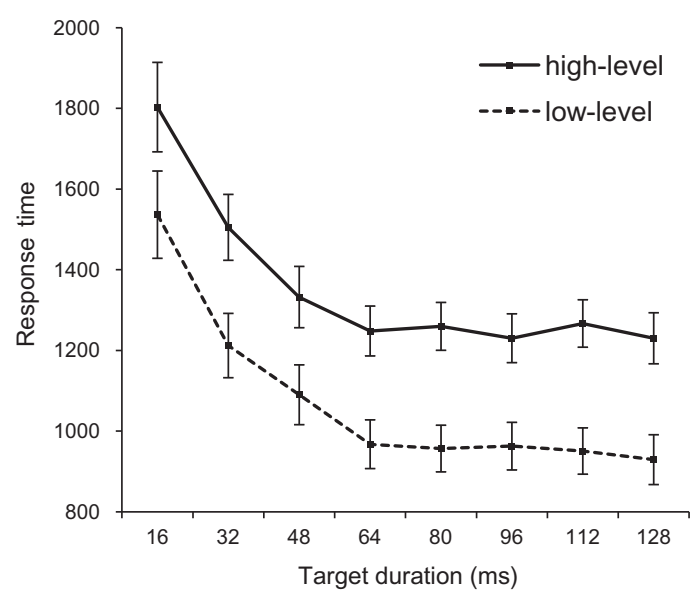

Experiment 1

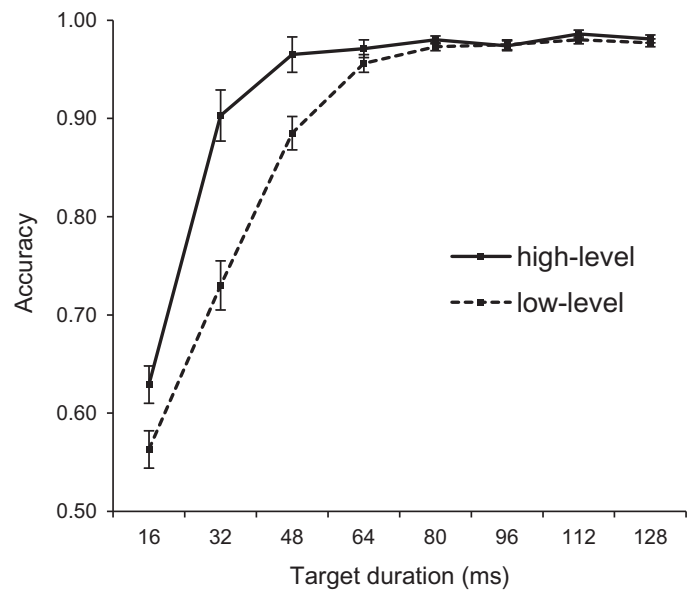

Experiment 2

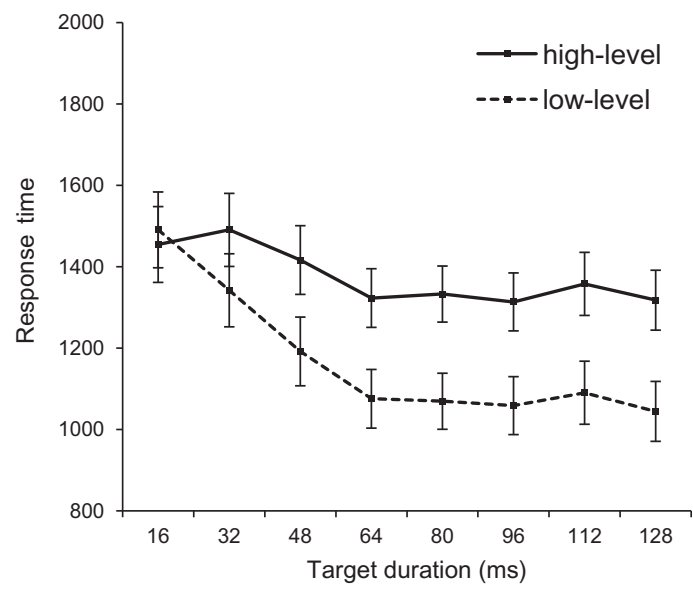

Experiment 2

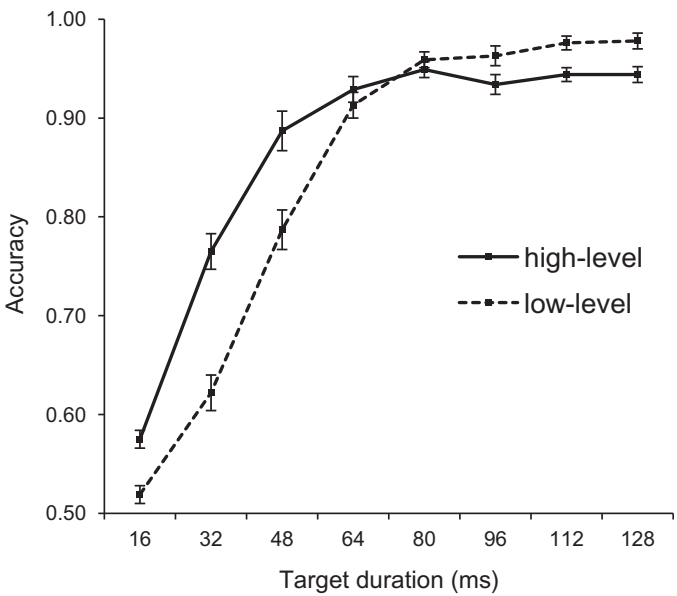

Fig. 2. Response time and accuracy in each condition of Experiment 1 (left) and Experiment 2 (right). 
see Fig. 2, and the accuracy results below). The main effects of LoP, $F(1,50)=3.94, p=.05, \eta_{p}{ }^{2}=.07$, and Target Duration, $F(7,350)=24.46, p<.0001, \eta_{p}{ }^{2}=.33$, as well as the LoP $\times$ Target Duration interaction, $F(7,350)=5.27, p=.005, \eta_{p}{ }^{2}=.09$, were significant. However, when tested without the $16 \mathrm{~ms}$ target duration condition, the interaction was no longer significant, $F(6,300)=1.5, p=.22, \eta_{p}{ }^{2}=.03$ (replicating the results of E1), while the main effect of Target Duration remained significant, $F(6,300)=23.85, p<.0001, \eta_{p}{ }^{2}=.32$, and the main effect of LoP slightly increased (1364 ms in high-level task vs $1124 \mathrm{~ms}$ in low-level task), $F(1,50)=5.32, p=.025, \eta_{p}{ }^{2}=.10$. To conclude, in RT measurement, the LoP effect was independent from Target Duration.

\subsubsection{Accuracy}

Experiment 1: Both main effects were significant: Target Duration, $F(7,273)=274.15, p<.001, \eta_{p}{ }^{2}=.87$, and LoP, $F(1,39)=13.85, p=.001, \eta_{p}{ }^{2}=.26$. The LoP $\times$ Target Duration interaction was also significant, $F(7,273)=13.72, p<.001$, $\eta_{p}{ }^{2}=.26$, reflecting the fact that the high-level task was performed significantly better than the low-level task when target duration was $32 \mathrm{~ms}, t(39)=4.77, p<.006$, and $48 \mathrm{~ms}, t(39)=3.24, p<.006$.

Experiment 2: In the second experiment, both main effects were significant: Target Duration, $F(7,350)=549.83, p<.001$, $\eta_{p}{ }^{2}=.91$, and LoP, $F(1,50)=4.14, p=.047, \eta_{p}{ }^{2}=.07$, along with the LoP $\times$ Target Duration interaction, $F(7,350)=25.78$, $p<.001, \eta_{p}{ }^{2}=.34$. As in $\mathrm{E} 1$, the high-level task was performed significantly better than the low-level task when target durations were short: $16 \mathrm{~ms}, t(50)=4.35, p<.006,32 \mathrm{~ms}, t(50)=5.52, p<.006$, and $48 \mathrm{~ms}, t(50)=3.60, p<.006$. However, the effect was reversed for the two longest target durations, 112 and $128 \mathrm{~ms}$, respectively, $t(50)=3.21, p<.006$, and $t(50)=3.01, p<.006$. The LoP effects for the intermediate target durations were not significant, $t$ 's $(50) \leqslant 1.9$ (for the detailed results, see: Table A.1 in Appendix A). Summarizing, LoP effects were visible not only in RT's, but also in accuracy measurement.

3.1.2.1. Accuracy in the trials with $16 \mathrm{~ms}$ target duration. In both experiments, accuracy was lowest in the trials with $16 \mathrm{~ms}$ target duration, thus we tested if it was still better than chance (i.e., 50\%). In E1, accuracy was above chance level for both the low-level task (56\%), $t(20)=3.16, p=.005$, and the high-level task $(62 \%), t(19)=7.01, p<.001$. The difference between the levels was significant, $t(39)=2.42, p=.02$. In E2, the accuracy in the low-level task was only $2 \%$, but still significantly above chance level, $t(25)=2.58, p=.016$. In the high-level task, accuracy was $57 \%, t(19)=7.23, p<.001$, and the difference between the levels was also significant, $t(50)=4.35, p<.001$.

\subsection{Perceptual Awareness Scale (PAS)}

\subsubsection{PAS ratings and accuracy}

The relationship between accuracy and PAS ratings was analyzed by means of logistic mixed model with LoP (2 levels), Awareness Rating (4 levels) and Target Duration (8 levels), all possible interactions, and random subject specific intercept. The PAS ratings were significantly related to accuracy in each condition of both experiments. We found significant slope effects for target durations and both LoP groups in E1, $z$ 's $\geqslant 7.07$. $p$ 's $<.001$, and E2, $z^{\prime} \mathrm{s} \geqslant 7.16$. $p$ 's $<.001$, except the 16 ms target duration condition of the low-level task of $\mathrm{E} 2, z=1.50 ; p=.13$, which corresponded to very low (close to chance level) accuracy in this condition. Additionally, we found that in E2 the relation between visibility ratings and accuracy was stronger in the high-level than in the low-level condition for the shortest durations, i.e., 16 and $32 \mathrm{~ms}$, while the opposite was observed for the longest duration (128 ms).

\subsubsection{PAS rating distribution}

The PAS rating distributions (see Fig. 3 ) showed that in the trials with longer (i.e., optimal for conscious perception) target durations ( $\geqslant 64 \mathrm{~ms}$ ), participants used the highest PAS point (4) more frequently than in those with shorter durations, indicating clear target visibility in these conditions. Conversely, in the trials with shorter target durations $(16,32,48 \mathrm{~ms})$, the middle PAS ratings were more frequently used. As could be expected, in the trials with the shortest target duration $(16 \mathrm{~ms})$, the lowest PAS point (1) was used most frequently, indicating no visibility of the targets. The question of interest was, however, whether the middle ratings were used more frequently in the low-level task than in the high-level task, as the LoP hypothesis would predict.

To test this prediction, we calculated a PAS middle rating frequency index, which tallies the frequency of middle ratings (2 and 3 ) in each condition of both experiments. We assumed that more frequent usage of the middle ratings reflects more graded visibility (interpreted as the intermediate step between conscious and unconscious perception, cf. Sandberg et al., 2010; Sergent \& Dehaene, 2004; Wierzchoń et al., 2014; Windey et al., 2014), whereas less frequent usage of those ratings reflects less graded visibility. Next, we tested the differences between the indexes obtained in the low-level and in the high-level tasks (as depicted in Fig. 4) by means of mixed logistic model with LoP (2 levels) and Target Duration (8 levels), their interactions, and random subject specific intercept. Table 1 presents LoP effects within each presentation time for both experiments.

Experiment 1. The overall difference between the extreme and middle ratings for high and low-level tasks was not significant, but LoP interacted with Target Duration. Crucially, in line with the LoP hypothesis, the middle ratings were used more frequently when the target duration was $48 \mathrm{~ms}, z=2.72, p<.01$, and $64 \mathrm{~ms}, z=2.06, p<.05$. Differences between the highand low-level tasks in the other target durations were not significant. 

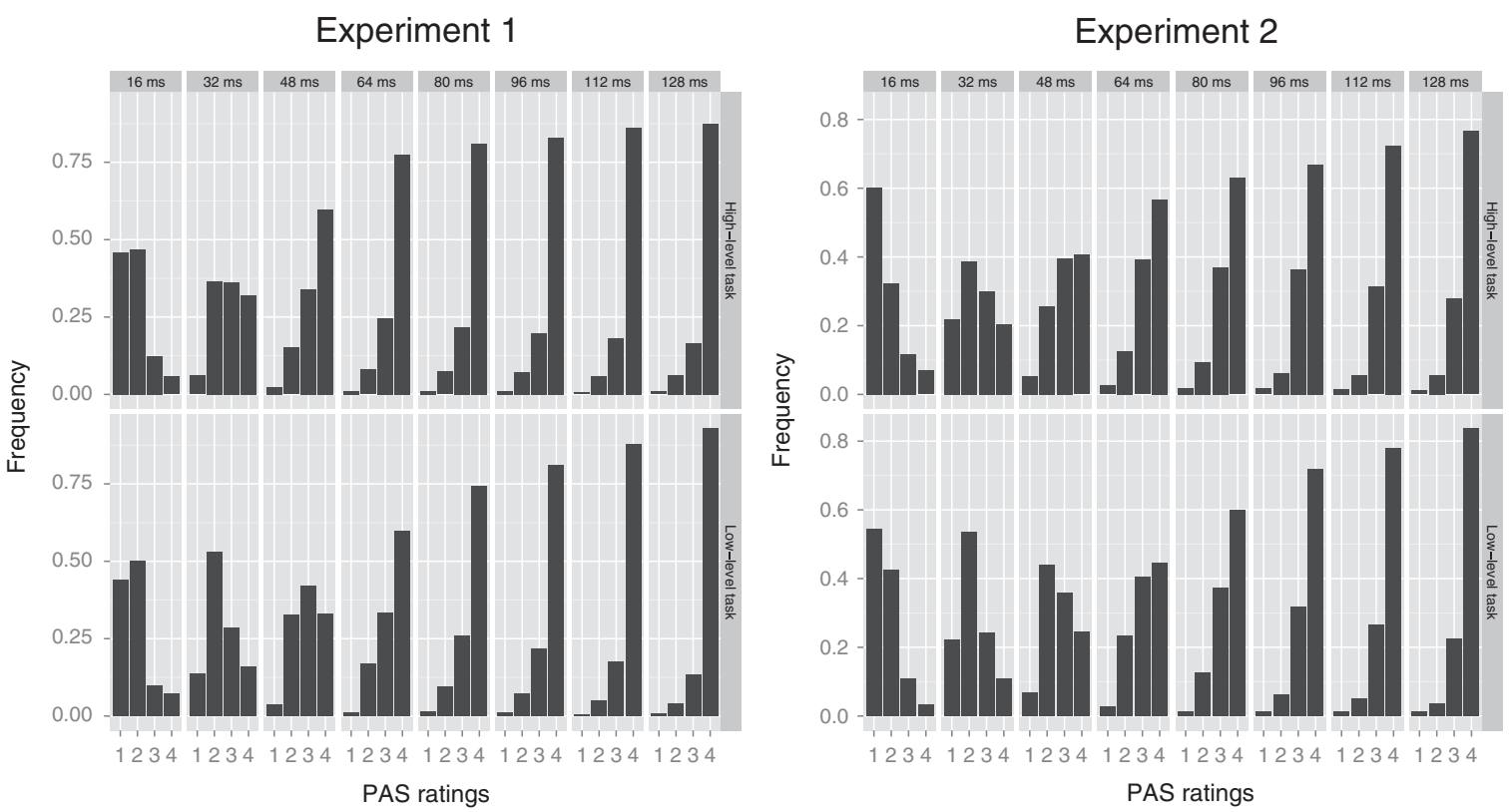

Fig. 3. PAS rating distributions in the low- and high-level tasks in Experiment 1 (left panel) and Experiment 2 (right panel).

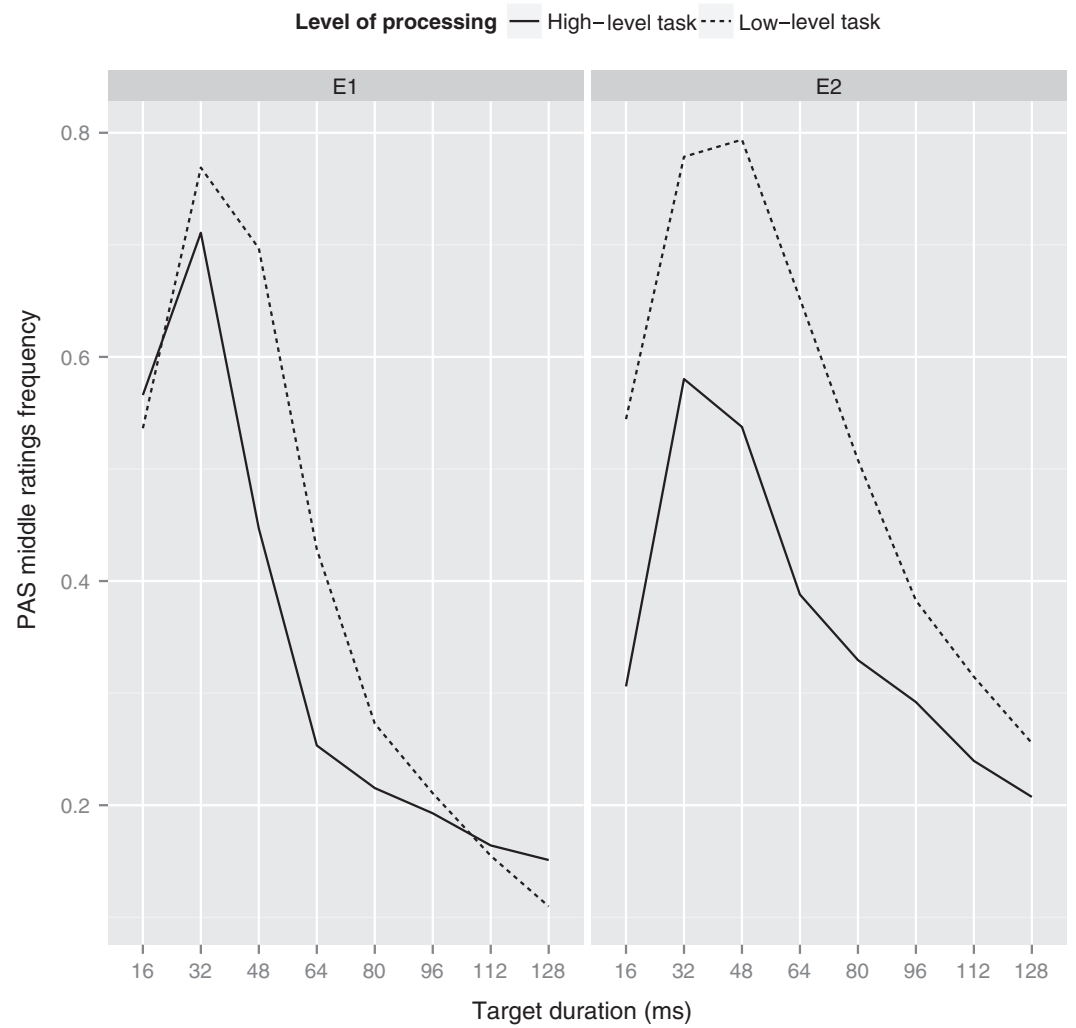

Fig. 4. The indexes of usage of the PAS middle ratings ( 2 and 3 ) for the low-level task (dashed lines) and the high-level task (solid lines) of Experiment 1 (left) and Experiment 2 (right).

Experiment 2. When the low-level task was the color discrimination task (instead of the letter case identity task in E1), more pronounced effects of LoP were obtained. The middle ratings were, in general, used more frequently in the low-level task than in the high-level task, $z=6.56, p<.001$, while being most pronounced for the $32 \mathrm{~ms}, z=9.08, p<.001$, and $48 \mathrm{~ms}, z=8.35, p<.001$, durations, which corresponds to the results of E1. 
Table 1

LoP effects on frequencies of PAS middle ratings usage, analyzed by means of mixed logistic regression model with LoP (2 levels) and Target Duration (8 levels) as factors. To improve readability, LoP was nested within target durations.

\begin{tabular}{|c|c|c|c|c|c|c|c|c|}
\hline & \multicolumn{4}{|c|}{$\begin{array}{l}\text { Experiment } 1 \\
N=41, \# \text { observations: } 26,221\end{array}$} & \multicolumn{4}{|c|}{$\begin{array}{l}\text { Experiment } 2 \\
N=50, \# \text { observations: } 32,553\end{array}$} \\
\hline & Estimate & SE & $z$ & $p$ & Estimate & SE & $z$ & $p$ \\
\hline \multicolumn{9}{|c|}{ Effect of LoP on middle ratings probability } \\
\hline $16 \mathrm{~ms}$ & -.12 & 0.38 & -.31 & .76 & 1.00 & .12 & 7.69 & $<.001^{* * *}$ \\
\hline $32 \mathrm{~ms}$ & .30 & 0.39 & .79 & .43 & .93 & .13 & 7.07 & $<.001^{* * *}$ \\
\hline $48 \mathrm{~ms}$ & 1.05 & 0.38 & 2.72 & $<.01^{* *}$ & 1.20 & .13 & 9.08 & $<.001^{* * * *}$ \\
\hline $64 \mathrm{~ms}$ & .79 & 0.39 & 2.06 & $<.05^{*}$ & 1.08 & .13 & 8.35 & $<.001^{* * *}$ \\
\hline $80 \mathrm{~ms}$ & .31 & 0.39 & .81 & .42 & .74 & .13 & 5.73 & $<.001^{* * *}$ \\
\hline $96 \mathrm{~ms}$ & .11 & 0.39 & .29 & .77 & .41 & .13 & 3.12 & $<.01^{* *}$ \\
\hline $112 \mathrm{~ms}$ & -.07 & 0.39 & -.18 & .86 & .38 & .13 & 2.85 & $<.01^{* *}$ \\
\hline \multirow[t]{2}{*}{$128 \mathrm{~ms}$} & -.37 & 0.39 & -.94 & .35 & .27 & .13 & 2.02 & $<.05^{*}$ \\
\hline & \multicolumn{4}{|c|}{$\begin{array}{l}\text { Likelihood ratio } \chi^{2}(16)=4563, p<.001 \\
{ }^{*} p<.05 \\
{ }^{* *} p<.01 \\
{ }^{* * *} p<.001\end{array}$} & \multicolumn{4}{|c|}{$\begin{array}{l}\text { Likelihood ratio } \chi^{2}(16)=3217, p<.001 \\
{ }^{*} p<.05 \\
{ }^{* *} p<.01 \\
{ }^{* * *} p<.001\end{array}$} \\
\hline
\end{tabular}

In order to examine whether the above effects were not artifacts produced by differences in accuracy between conditions, we performed a similar logistic mixed model analysis with LoP and Target Duration, while controlling for accuracy. In both experiments, the overall pattern of results was the same as in the first analyses - all the effects remained significant (E1: $z$ 's $\geqslant 1.77, p$ 's $\leqslant .07 ; \mathrm{E} 2: z^{\prime} \mathrm{s} \geqslant 3.42, p$ 's $<.001$ ), clearly demonstrating that the differences between the low- and high-level tasks in PAS rating distributions were not caused by differences in accuracy.

To summarize, in both experiments the PAS rating distributions were in line with the LoP hypothesis.

\section{Discussion}

\subsection{Levels of processing in the objective measures of performance}

Does gradualness of the transition from unconscious to conscious vision depend on the level of processing? Here, we tested the LoP hypothesis using two variants of a task, which was designed specifically to differentiate between low and high levels of processing. The two experiments demonstrated very similar effects of LoP in terms of response times. Namely, in the high-level tasks, RT were longer than in the low-level tasks, revealing the classic LoP effect (Posner \& Mitchell, 1967). We may therefore assume that the high-level task imposed processing of the stimuli at a higher, semantic level, or, using Craik and Lockhart's metaphor, at a deeper level of the cognitive hierarchy. It is worth mentioning that such an interpretation is also in line with several neuroimaging studies, which showed correlations between levels of processing imposed by a task demands and the observed level of cortical activation (e.g., Kapur et al., 1994; Otten \& Rugg, 2001; Petersen et al., 1988).

The LoP effect on RT was independent of target duration. However, the accuracy analysis showed an interesting interaction between LoP and target duration. When stimulus duration was long enough to enable clear perception of the targets (i.e., 122 and $128 \mathrm{~ms}$ ), performance tended to be better in the low-level task than in the more demanding high-level task, matching the RT results. ${ }^{2}$ However, when target duration was shorter, that is, when it was suboptimal for conscious perception $(16,32$, and $48 \mathrm{~ms})$, accuracy was better in the high-level tasks, while RT was still longer in these conditions when compared to the low-level task. The latter result seems counterintuitive, as more demanding high-level tasks should be performed with lower accuracy than easier, low-level tasks, especially in conditions that involve short presentation durations. However, it is possible that the longer RT in the high-level task provided more time for the accumulation of sensory evidence, thereby contributing to better accuracy. ${ }^{3}$ The higher accuracy in the high-level tasks with suboptimal target durations might also be interpreted as a speed-accuracy trade-off because being more conservative in the high-level tasks would result in longer RT and higher accuracy. In this case, however, the RT differences between the two LoP conditions at the target durations that are optimal for conscious perception should no longer be observed, as there is rather no reason to expect such a trade-off when the target is clearly visible. Also, in the case of a speed-accuracy trade-off, RT effects should interact with increasing task difficulty (i.e., the more difficult the task, the slower the responses, to maintain high accuracy with increasing difficulty). However, the LoP effects in RT were independent of target durations, which suggests that the interaction between target duration and LoP in accuracy may be relatively independent from the LoP effect in RT. In other words, the LoP effect may have different sources.

An alternative explanation of higher accuracy in high LoP condition for short stimulus durations would be that top-down processing makes it possible to partially overcome the shortage of perceptual information stemming from suboptimal

\footnotetext{
${ }^{2}$ Note that this LoP effect for accuracy was observed only in E2, due to a ceiling effect in E1.

${ }^{3}$ It is worth noting that higher accuracy for the high-level task than the low-level task is in line with the results obtained by Windey et al. (2014).
} 
stimulus duration. ${ }^{4}$ Evidence from human single-cell recordings and computational studies suggests that high-level, semantic representations are sparse, abstract, and invariant, that is, largely independent from the exact form of physical stimulation (Quiroga, Kreiman, Koch, \& Fried, 2008; Waydo \& Koch, 2008; see also the predictive coding theory, e.g., Panichello, Cheung, \& Bar, 2012). Thus, we may speculate that semantic or more abstract representations might be built in a more top-down manner, thereby requiring less amount of bottom-up sensory information. If so, the lack of precise perceptual evidence about stimuli would be less problematic or less disturbing when the tasks require more abstract, semantic processing of stimuli (but such processing might still take more time, than the low-level tasks, which is exactly what we observed).

To summarize, both experiments have shown the expected LoP effects, which provides a solid ground for the interpretation of differences in the gradual nature of conscious perception in terms of depth of processing, as proposed by Windey et al. (2013). The study also revealed that the effects of LoP on accuracy may depend on whether the presentation of stimuli is optimal (sufficiently long) or suboptimal (too short) for conscious perception.

\subsection{Relationship between accuracy and stimulus visibility}

The correlation between accuracy and stimulus visibility was high in both experiments, as expected (cf. Wierzchoń et al., 2014). Additionally, in the second experiment, we found that when stimuli were presented very briefly ( 16 and $32 \mathrm{~ms}$ of target durations), accuracy proved to be increasing more gradually in the low-level task than in the high-level task. This result replicates the findings of Windey et al. (2014), and is congruent with the LoP hypothesis. The inverse effect was observed for the longest duration (128 ms) of E2. Here, accuracy was increasing more gradually in the high-level than the low-level task, however, it seems that this effect might arise due to a ceiling effect in accuracy. It is worth noting that in the study by Windey et al. (2013) accuracy did not simply increase faster in the high-level task. In fact, it increased more slowly for short stimulus durations and faster for long stimulus durations. This result has not been replicated in the experiments discussed in this paper. This discrepancy might arise from the fact that the processing time varied across these studies, i.e., in the experiments presented in this paper clear differences in RT between the low- and high-level conditions were observed, while in the paper by Windey et al. (2013) no LoP effects in RT were reported. As we suggested in the previous section (4.1), the counterintuitive effect of higher accuracy in the high LoP condition might be due to a speed-accuracy trade-off.

\subsection{Stimulus visibility and the level of processing hypothesis}

According to the LoP hypothesis, information processing at a lower level is associated with a more graded transition from unconscious to conscious perception than processing at a higher level (Windey \& Cleeremans, 2015; Windey et al., 2013). Therefore, we expected a higher frequency of the PAS middle ratings usage in the low-level task than in the high-level task. The results of Experiment 1 show that when target duration was short (48 and $64 \mathrm{~ms}$ - conditions with moderate levels of accuracy), the middle PAS ratings were used more often in the low-level task than in the corresponding conditions of the high-level task. This result supports the idea that the transition between unconscious and conscious processing is more gradual when the task involves a low-level processing. The second experiment showed similar, but more pronounced effects of LoP on stimulus visibility. The middle PAS ratings were again used more frequently in the low-level task than in the high-level task, and the effect was significant for all target durations. Still, the effect was most evident around the threshold for conscious perception (i.e., for 48 and $64 \mathrm{~ms}$ target durations), and slightly decreased when the stimuli were presented either with short $(<48 \mathrm{~ms}$ ) or long durations $(>64 \mathrm{~ms})$, congruently with the results of Experiment 1 . This interaction with target duration seems to be a result of the floor and ceiling effects on target visibility, which appear to override more subtle effects of LoP on PAS ratings.

We can speculate on the underlying neural mechanism that drives the more gradual transition during low-level processing. Previous studies have found correlations between conscious perception of low-level visual stimuli and early recurrent processing in visual cortex (Boehler, Schoenfeld, Heinze, \& Hopf, 2008; Fahrenfort, Scholte, \& Lamme, 2008). These recurrent interactions are proposed to be associated with conscious visual experience through processes such as figure-ground segregation and perceptual organization (Koivisto \& Silvanto, 2011; Lamme, 2010). Future neuroimaging studies could specifically target posterior brain regions to investigate whether the gradual transition from unconscious to conscious processing for low-level stimuli and tasks (as presented in this paper) is associated with or caused by a gradual increase in recurrent processing between early visual regions.

\subsection{Alternative interpretations}

While the present results are in line with the LoP hypothesis, they nevertheless do not completely make it possible to rule out alternative interpretations of the differences in visibility of stimuli in the low- and high-level tasks. One such alternative explanation would be that processing of information at higher levels inherently imposes a longer processing time (reflecting in longer RT), and thereby enables more time for the accumulation of evidence necessary to elaborate a clear, detailed conscious representation. In other words, in the low-level task, representation at the very moment of responding involves a

\footnotetext{
${ }^{4}$ This does not imply that participants were not aware of the stimuli.
} 
Table A.1

Results of logistic mixed models with LoP, Awareness Rating, and Target Duration. Separate intercepts and slopes parameterization were used to improve the readability of regression coefficients. Part A of the table shows the scale slopes for different target durations separately for the low- and high-level tasks. Part B of the table shows the interactions between level and slopes within time. To improve readability, only the slope estimates are shown.

\begin{tabular}{|c|c|c|c|c|c|c|c|c|}
\hline & \multicolumn{4}{|c|}{$\begin{array}{l}\text { Experiment } 1 \\
N=41 \text {, \# observations: } 26,166\end{array}$} & \multicolumn{4}{|c|}{$\begin{array}{l}\text { Experiment } 2 \\
N=50, \# \text { observations: } 32,398\end{array}$} \\
\hline & Estimate & SE & $z$ & $p$ & Estimate & SE & $z$ & $p$ \\
\hline \multicolumn{9}{|c|}{ A. PAS rating slopes within conditions } \\
\hline LoP low, 16 ms: rating & .58 & .07 & 8.15 & $<.001^{* * * *}$ & .09 & .06 & 1.50 & .13 \\
\hline LoP low, 32 ms: rating & .97 & .08 & 12.19 & $<.001^{* * *}$ & .42 & .06 & 7.21 & $<.001^{* * *}$ \\
\hline LoP low, 48 ms: rating & 1.23 & .11 & 11.63 & $<.001^{* * *}$ & .87 & .07 & 11.81 & $<.001^{* * *}$ \\
\hline LoP low, 64 ms: rating & 2.02 & .18 & 11.25 & $<.001^{* * *}$ & 1.15 & .10 & 11.18 & $<.001^{* * *}$ \\
\hline LoP low, $80 \mathrm{~ms}$ : rating & 2.08 & .19 & 10.91 & $<.001^{* * *}$ & 1.62 & .15 & 11.02 & $<.001^{* * *}$ \\
\hline LoP low, 96 ms: rating & 1.85 & .18 & 10.41 & $<.001^{* * *}$ & 1.43 & .14 & 10.07 & $<.001^{* * *}$ \\
\hline LoP low, 112 ms: rating & 1.63 & .19 & 8.43 & $<.001^{* * *}$ & 1.76 & .16 & 10.70 & $<.001^{* * *}$ \\
\hline LoP low, 128 ms: rating & 2.14 & .19 & 11.04 & $<.001^{* * *}$ & 2.22 & .19 & 11.41 & $<.001^{* * *}$ \\
\hline LoP high, 16 ms: rating & .54 & .08 & 7.15 & $<.001^{* * *}$ & .40 & .06 & 7.16 & $<.001^{* * *}$ \\
\hline LoP high, 32 ms: rating & 1.07 & .11 & 9.73 & $<.001^{* * *}$ & .83 & .06 & 13.06 & $<.001^{* * *}$ \\
\hline LoP high, 48 ms: rating & 1.46 & .16 & 9.19 & $<.001^{* * *}$ & .98 & .09 & 11.44 & $<.001^{* * *}$ \\
\hline LoP high, 64 ms: rating & 1.79 & .18 & 10.04 & $<.001^{* * *}$ & 1.43 & .11 & 12.60 & $<.001^{* * *}$ \\
\hline LoP high, $80 \mathrm{~ms}$ : rating & 1.63 & .19 & 8.48 & $<.001^{* * *}$ & 1.38 & .13 & 10.97 & $<.001^{* * *}$ \\
\hline LoP high, 96 ms: rating & 1.55 & .17 & 9.10 & $<.001^{* * *}$ & 1.26 & .12 & 10.77 & $<.001^{* * *}$ \\
\hline LoP high, $112 \mathrm{~ms}$ : rating & 1.64 & .23 & 7.07 & $<.001^{* * *}$ & 1.53 & .12 & 12.414 & $<.001^{* * *}$ \\
\hline LoP high, $128 \mathrm{~ms}$ : rating & 1.79 & .20 & 8.87 & $<.001^{* * *}$ & 1.47 & .13 & 11.665 & $<.001^{* * *}$ \\
\hline \multicolumn{9}{|c|}{ B. PAS ratings slopes $\times$ LoP within time conditions } \\
\hline LoP $\times$ ratings at $16 \mathrm{~ms}$ & .04 & .10 & .35 & .72 & -.31 & .08 & -3.791 & $<.001^{* * *}$ \\
\hline LoP $\times$ ratings at $32 \mathrm{~ms}$ & -.11 & .14 & -.78 & .44 & -.42 & .09 & -4.850 & $<.001^{* * *}$ \\
\hline LoP $\times$ ratings at $48 \mathrm{~ms}$ & -.23 & .19 & -1.22 & .22 & -.11 & .11 & -0.940 & .35 \\
\hline LoP $\times$ ratings at $64 \mathrm{~ms}$ & .23 & .25 & .90 & .37 & -.28 & .15 & -1.843 & .07 \\
\hline LoP $\times$ ratings at $80 \mathrm{~ms}$ & .45 & .27 & 1.67 & .09 & .24 & .19 & 1.238 & .22 \\
\hline LoP $\times$ ratings at $96 \mathrm{~ms}$ & .30 & .25 & 1.21 & .23 & 0.17 & .18 & 0.906 & .36 \\
\hline LoP $\times$ ratings at $112 \mathrm{~ms}$ & -.01 & .30 & -.04 & .96 & 0.23 & .21 & 1.105 & .27 \\
\hline \multirow[t]{2}{*}{ LoP $\times$ ratings at $128 \mathrm{~ms}$} & .35 & .28 & 1.27 & .21 & 0.74 & .23 & 3.201 & $<.01^{* * *}$ \\
\hline & \multicolumn{4}{|c|}{$\begin{array}{l}\text { Likelihood ratio } \chi^{2}(32)=5530, p<.001 \\
{ }^{*} p<.05 \\
{ }^{* *} p<.01 \\
{ }^{* * *} p<.001\end{array}$} & \multicolumn{4}{|c|}{$\begin{array}{l}\text { Likelihood ratio } \chi^{2}(32)=7216, p<.001 \\
{ }^{*} p<.05 \\
{ }^{* *} p<.01 \\
{ }^{* * *} p<.001\end{array}$} \\
\hline
\end{tabular}

smaller amount of evidence because the response is given after a shorter processing time than in the high-level task. Nevertheless, it is also plausible that these two aspects, level and duration of processing, are just two sides of the same coin. Namely, imposing processing of information at higher levels might always enforce longer time of processing (cf. the mental chronometry argument presented in the Introduction). If this is the case, then the LoP hypothesis might be correct exactly because higher levels of processing tend to be systematically associated with longer processing time.

Finally, it seems worth mentioning that in the high-level tasks the frequency of the middle ratings was still relatively high. This seems to suggest that subjective judgments about conscious experience might be generally graded (cf. Nieuwenhuis \& de Kleijn, 2011; Overgaard et al., 2006), rather than dichotomous (as it was suggested by Sergent \& Dehaene, 2004). ${ }^{5}$ Note that the frequency of the lowest PAS ratings was very low in all conditions (presumably reflecting conscious processing of stimuli). Thus, the effect of LoP appears to originate mainly from the frequency of the middle (2 and 3$)$ and the highest (4) ratings. It might be that what differs between the LoP conditions is the threshold at which participants begin to use the highest rating. We might thus speculate why we observe such systematic differences in rating frequencies. It seems plausible that this might be related to the diversity of the mechanisms underlying subjective reports. Particularly, the systematic differences in rating frequencies might be related to two, partially independent mechanisms. The first one (e.g., the accumulation of evidence, as proposed above) might influence the gradedness of access to the first-order representation (access to the representation of stimuli in the cognitive system, see: hierarchical models of consciousness, Lau \& Rosenthal, 2011). The second mechanism might be related to participants' subjective judgments of that first-order accessibility. Our subjective judgments usually operate on a metacognitive level of representations (see e.g., Seth, 2008). It thus seems possible that the effects of LoP are in fact restricted to the first-order representation, and then higher-order judgments provide a more complex, and thus more gradual interpretation of a dichotomous access to the first-order representation. Hence, the real dichotomy (if it indeed exists) would be observable only at the first-order level, whereas the subjective judgments (generated by higher-order representation) would always be gradual. However, we still observed significantly more graded ratings in the low-level task than in

\footnotetext{
${ }^{5}$ Recently, Nieuwenhuis and de Kleijn (2011) have reported a gradual pattern of awareness ratings even in tasks that were very similar to the ones used in Sergent and Dehaene's (2004) study (i.e., rapid serial visual presentation tasks, with words or letters as stimuli). This result suggests that some factors, other than LoP, may affect the gradualness of conscious perception as well.
} 
the high-level task. Thus, it seems that differences in first-order access may be reflected to some extent in the higher-order subjective judgments.

\subsection{Conclusion}

In this paper, we provide further evidence suggesting that the level of processing affects the transition from unconscious to conscious perception. The two experiments demonstrated that subjective visibility of stimuli is rated as clear more frequently in the high-level task than in the low-level task, indicating that the transition from unconscious to conscious perception might be less gradual when a task imposes processing of stimuli at higher (or deeper) levels of the cognitive system. To conclude, the results are fully in agreement with the LoP hypothesis (Windey et al., 2013), although some alternative interpretations still remain possible.

\section{Acknowledgments}

A.A. and M.W. were supported by SONATA BIS grant from the National Science Centre of Poland (2012/07/E/HS6/01037). D.A. was supported by SONATA grant from the National Science Centre of Poland (2012/05/D/HS6/03363). B.W. is supported by a fellowship from the National Fund for Scientific Research (FRS - FNRS Belgium), and by a scholarship from the Fyssen Foundation. A.C. is a Research Director with the F.R.S. FNRS (Belgium). His work is supported by ERC Advanced grant "RADICAL" and by Interuniversity Poles of Attraction grant P7/33 from the Belgian Science Policy Office (BELSPO).

\section{Appendix A}

See Table A.1.

\section{References}

Asplund, C. L., Fougnie, D., Zughni, S., Martin, J. W., \& Marois, R. (2014). The attentional blink reveals the probabilistic nature of discrete conscious perception. Psychological Science, 25(3), 824-831.

Boehler, C., Schoenfeld, M., Heinze, H., \& Hopf, J. (2008). Rapid recurrent processing gates awareness in primary visual cortex. Proceedings of the National academy of Sciences of the United States of America, 105, 8742-8747.

Cleeremans, A. (2011). The radical plasticity thesis: How the brain learns to be conscious. Frontiers in Psychology, 2(86), 1-12.

Craik, F. M., \& Lockhart, R. S. (1972). Levels of processing: A framework for memory research. Journal of Verbal Learning and Verbal Behaviour, 11, 671-684.

Dehaene, S., Changeux, J. P., \& Naccache, L. (2011). The global neuronal workspace model of conscious access: From neuronal architectures to clinical applications. In S. Dehaene \& Y. Christen (Eds.), Characterizing consciousness: From cognition to the clinic? (pp 55-84). London, New York: Springer-Verlag.

Dehaene, S., \& Cohen, L. (2011). The unique role of the visual word form area in reading. Trends in Cognitive Sciences, 15(6), $254-262$.

del Cul, A., Baillet, S., \& Dehaene, S. (2007). Brain dynamics underlying the nonlinear threshold for access to consciousness. PLoS Biology, 5, e260.

Fahrenfort, J. J., Scholte, H. S., \& Lamme, V. A. F. (2008). The spatiotemporal profile of cortical processing leading up to visual perception. Journal of Vision, $8(1), 1-12$.

Forster, K. I., \& Forster, J. C. (2003). DMDX: A windows display program with millisecond accuracy. Behavior Research Methods, Instruments, E` Computers, 35, $116-124$.

Gaillard, R., Dehaene, S., Adam, C., Clémenceau, S., Hasboun, D., Baulac, M., et al (2009). Converging intracranial markers of conscious access. PLoS Biology, 7 , e61.

Garaizar, P., Vadillo, M. A., López-de-Ipiña, D., \& Matute, H. (2014). Measuring software timing errors in the presentation of visual stimuli in cognitive neuroscience experiments. PLoS One, 9(1), e85108.

Kapur, S., Craik, F. I., Tulving, E., Wilson, A. A., Houle, S., \& Brown, G. M. (1994). Neuroanatomical correlates of encoding in episodic memory: Levels of processing effect. Proceedings of the National academy of Sciences of the United States of America, 91, 2008-2011.

Koivisto, M., \& Silvanto, J. (2011). Relationship between visual binding, reentry and awareness. Consciousness and Cognition, $20,1293-1303$.

Lamme, V. A. F. (2010). How neuroscience will change our view on consciousness. Cognitive Neuroscience, 1, $204-220$.

Lau, H., \& Rosenthal, D. (2011). Empirical support for higher-order theories of conscious awareness. Trends in Cognitive Sciences, 15(8), 365-373.

Lockhart, R. S., \& Craik, F. I. M. (1990). Levels of processing: A retrospective commentary on a framework for memory research. Canadian Journal of Psychology, 44(1), 87-112.

Nieuwenhuis, S., \& de Kleijn, R. (2011). Consciousness of targets during the attentional blink: A gradual or all-or-none dimension? Attention, Perception, E' Psychophysics, 73(2), 364-373.

Otten, L. J., \& Rugg, M. D. (2001). Task-dependency of the neural correlates of episodic encoding as measured by fMRI. Cerebral Cortex, 11, 1150-1160.

Overgaard, M., Rote, J., Mouridsen, K., \& Ramsøy, T. (2006). Is conscious perception gradual or dichotomous? A comparison of report methodologies during a visual task. Consciousness and Cognition, 15, 700-708.

Panichello, M. F., Cheung, O. S., \& Bar, M. (2012). Predictive feedback and conscious visual experience. Frontiers in Psychology, 3, 620.

Petersen, S. E., Fox, P. T., Posner, M. I., Mintun, M., \& Raichle, M. E. (1988). Positron emission tomographic studies of the cortical anatomy of single-word processing. Nature, 331, 585-589.

Posner, M. I. (2005). Timing the brain: Mental chronometry as a tool in neuroscience. PLoS Biology, 3(2), $204-206$.

Posner, M. I., \& Mitchell, R. F. (1967). Chronometric analysis of classification. Psychological Review, 74, $392-409$.

Quiroga, Q. R., Kreiman, G., Koch, C., \& Fried, I. (2008). Sparse but not 'grandmother-cell' coding in the medial temporal lobe. Trends in Cognitive Science, 12, $87-91$.

Ramsøy, T. Z., \& Overgaard, M. (2004). Introspection and subliminal perception. Phenomenology and the Cognitive Sciences, 3(1), 1-23.

Sandberg, K., Timmermans, B., Overgaard, M., \& Cleeremans, A. (2010). Measuring consciousness: Is one measure better than the other? Consciousness and Cognition, 19, 1069-1078.

Sergent, C., \& Dehaene, S. (2004). Is consciousness a gradual phenomenon? Psychological Science, 15(11), 720-728.

Seth, A. K. (2008). Post-decision wagering measures metacognitive content, not sensory consciousness. Consciousness and Cognition, 17(3), 981-983.

Waydo, S., \& Koch, C. (2008). Unsupervised learning of individuals and categories from images. Neural Computation, 20(5), 1165-1178.

Wierzchoń, M., Paulewicz, B., Asanowicz, D., Timmermans, B., \& Cleeremans, A. (2014). Decisions in a visual identification task influence subsequent subjective stimulus awareness. Consciousness and Cognition, 27C, 109-120. 
Windey, B., \& Cleeremans, A. (2015). Consciousness as a graded and an all-or-none phenomenon: A conceptual analysis. Consciousness and Cognition, 35, 185-191.

Windey, B., Gevers, W., \& Cleeremans, A. (2013). Subjective visibility depends on level of processing. Cognition, 129(2), 404-409.

Windey, B., Vermeiren, A., Atas, A., \& Cleeremans, A. (2014). The graded and dichotomous nature of visual awareness. Philosophical Transactions of the Royal Society of London. Series B, Biological sciences, 369(1641), 20130282. 